bzw. die Oszillatorenstärke der schwingenden Elektronen und je geringer deren Anregungsenergie ist. Die letztere Bedingung ist besonders gut bei den typischen Farbstoffen mit ihren intensiven langwelligen Banden erfüllt, die erstere Bedingung wird durch die Aufreihung in geringen Abständen infolge der Ladungsneutralisation gegeben.

Der Metachromasie-Effekt durch Heparin ist also dem sog. Assoziations-Effekt wäßriger Farbstofflösungen analog. Es besteht nur der eine Unterschied, daß die Abstoßung der gleichgeladenen Farbstoff-Moll. im typischen Fall der Assoziation durch die starken Dipole des Wassers, also durch eine Wechselwirkung der Farbstoff-Moll. mit den Lösungsmittel-Moll. verhindert wird.

Unsere Deutung des Metachromasie-Effektes mit Heparin ist mit der von SylvéN ${ }^{19}$ in vielen Punkten übereinstimmend. SyLvÉn hat mit Azurblau, einem unsymmetrisch substituierten Thioninderivat, Wechselwirkungen zwischen diesem Farbstoff und verschiedenen chromotropen Substraten untersucht und kam ebenfalls zu der Ansicht, daß primär eine elektro.

19 Bengt Sylvén, Quart. J. microsc. Sci. [London] 95, 327 [1954]. statische Wechselwirkung zwischen Träger und Farbstoff die Aufreihung bzw. Anlagerung der FarbstoffMoll. auf Abstände von ungefähr $5 \AA$ verursacht. Erst sekundär soll die Bindung der Farbstoff-Moll. untereinander (er vermutet Wasserstoff- und Sauerstoff-Schwefelbindungen) die Ursache des Metachromasie-Effektes sein. Die Bandenverschiebung wird durch das Auftreten neuer Banden erklärt.

In diesem letzteren Punkt sind wir abweichender Meinung, da unsere Befunde eindeutig mit dem Assoziations-Effekt in wäßriger Lösung übereinstimmen und die Schwerpunktsverlagerung nach kurzen Wellen durch einen Intensitätsanstieg der Schwingungsbanden eines Elektronenübergangs verursacht wird. Für das Auftreten des Metachromasie-Effektes beim Acridinorange machen wir die Dispersionskräfte, die Überlappung der $\pi$-Elektronenwolken und eine daraus resultierende Verschiebung der Potentialkurven des Elektronenanregungs-Zustandes verantwortlich. Die Überlappung und Störung der $\pi$-Elektronensysteme kann erst bei einer Annäherung der Farbstoff-Moll. auf 4 bis $5 \AA$ erfolgen, da die Wirkungssphäre der $\pi$-Elektronen - oberhalb und unterhalb der Mol.-Ebene zusammen - auf diese Größe beschränkt ist.

\title{
NOTIZEN
}

\section{Über die Fähigkeit der Chlorella pyrenoidosa zur anaeroben Nitritreduktion}

Von Kurt Damaschke und Margot Lübke

Bundesanstalt für Materialprüfung, Berlin-Dahlem

(Z. Naturforschg. 13 b, 134-135 [1958]; eingeg. am 23. Dezember 1957)

Wie wir schon früher festgestellt haben ${ }^{1}$, besitzt auch Chlorella, ebenso wie Scenedesmus und Ankistrodesmus ${ }^{2}$ die Fähigkeit, nach langer Anaerobiose unter Stickstoff, Glucose zu Wasserstoff zu vergären. Ferner wurde von uns eine $\mathrm{CO}_{2}$-Reduktion bei Chlorella nachgewiesen, d. h. adaptierte Zellen haben die Fähigkeit, $\mathrm{CO}_{2}$ mit molekularem $\mathrm{H}_{2}$ im Licht zu reduzieren. Das deutet darauf hin, daß auch Chlorella ein HydrogenaseSystem besitzt. Daß dies von anderen Autoren nicht gefunden wurde, liegt wahrscheinlich daran, daß die Adaptationszeit, d. h. die Zeit der Anaerobiose, die zur Aktivierung des Hydrogenase-Systems notwendig ist, bei Chlorella sehr lang ist. Sie beträgt bei Scenedesmus

1 K. Damaschke, Z. Naturforschg. 12 b, 441 [1957].

2 H. Gaffron u. J. Rubin, J. gen. Physiol. 26, 219 [1942].

3 E. Kessler, Arch. Mikrobiol. 27, 166 [1957]. nur 2 Stdn., während es bei Chlorella etwa 4-5 Tage dauert, ehe die volle Aktivität erreicht ist. Um einen weiteren Beweis des Vorhandenseins eines HydrogenaseSystems in Chlorella zu erbringen, wurde die Fähigkeit der Zellen, in adaptiertem Zustand Nitrit zu reduzieren, untersucht. Wie KessLer ${ }^{3}$ gefunden hat, haben Algen, die ein Hydrogenase-System besitzen, die Fähigkeit, nach Adaptation Nitrit mit molekularem $\mathrm{H}_{2} \mathrm{zu}$ $\mathrm{NH}_{3}$ zu reduzieren. Die Nitrit-Reduktion ist der Hydrogenase-Aktivität proportional.

\section{Methodik}

Der $\mathrm{H}_{2}$ wurde elektrochemisch mit der $\mathrm{H}_{2} \mathrm{O}_{2}$-Meßmethode nach $\mathrm{W}_{\text {InKeLManN }}{ }^{4}$ gemessen. Als Meßelektrode diente eine platinierte Platin-Elektrode, als Gegenelektrode eine blanke Platinelektrode und als Vergleichselektrode eine $\mathrm{Hg} / \mathrm{Hg}_{2} \mathrm{SO}_{4}$-Elektrode. Das Potential wurde mit einem Potentiostaten nach Schwarz ${ }^{5}$ konstant gehalten. Mit dieser Anordnung kann man

${ }^{4}$ K. Damaschke u. D. Winkelmann, Z. Naturforschg. 12 b, 86 [1957].

5 W. Schwarz, Chemie-Ing.-Techn. 6, 423 [1956]. 
außer $\mathrm{H}_{2} \mathrm{O}_{2}$ noch eine Anzahl anderer reduzierender Substanzen messen, z. B. auch molekularen Wasserstoff ${ }^{1,6}$. Es wird nur der in Lösung befindliche Anteil angezeigt, so daß man unter Ausschluß der Gasphase arbeiten muß. Bei $25^{\circ} \mathrm{C}$ sind $78,5 \cdot 10^{-5} \mathrm{Mol} / l \mathrm{H}_{2}$ löslich.

Die Zellen wurden in Phosphatpuffer von $p_{\mathrm{H}} 5,4$ suspendiert. Die Zellkonzentration betrug $50 \mathrm{~mm}^{3}$ Zellen pro $\mathrm{cm}^{3}$ Lösung $(c=50)$.

Im Blindversuch wurde nachgewiesen, daß $\mathrm{KNO}_{2}$ keinen Einfluß auf die Elektroden ausübt.

\section{Versuche}

Nach einer bestimmten Adaptationszeit unter Sauerstoff-Ausschluß wurde molekularer Wasserstoff in die Zellsuspension geleitet. Nachdem sich ein konstanter Stromwert eingestellt hatte, wurden $1 \cdot 10^{-4}$ bzw. $2 \cdot 10^{-4} \mathrm{Mol} / l \mathrm{KNO}_{2}$ zugesetzt, und der $\mathrm{H}_{2}$-Verbrauch registriert. Die Versuche ergaben, daß die Geschwindigkeit des $\mathrm{H}_{2}$-Verbrauchs von der Adaptationszeit abhängig ist (Abb. 1 und Abb. 2). Bis zu einer Adaptations-

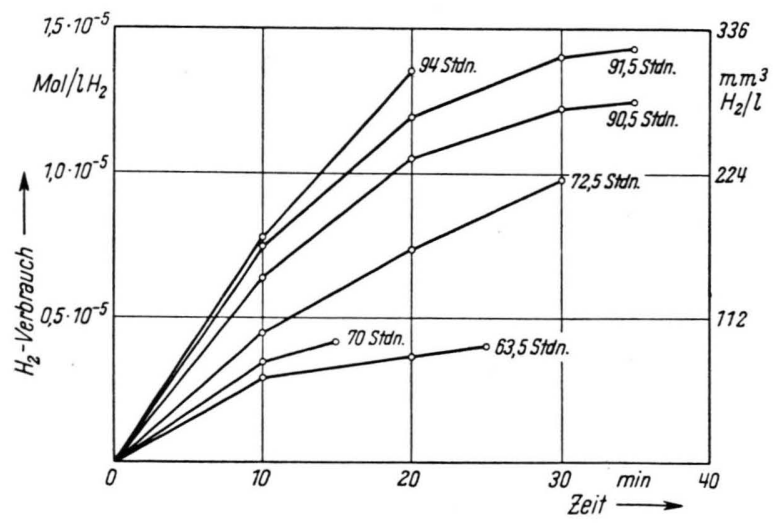

Abb. 1. $\mathrm{H}_{2}$-Verbrauch bei Nitritzugabe nach verschiedenen Adaptationszeiten. Nitritmenge jeweils $2 \cdot 10^{-4} \mathrm{Mol} / l \mathrm{KNO}_{2}$.

zeit von $50 \mathrm{Stdn}$. wird das zugesetzte Nitrit nicht reduziert. Nach dieser Zeit ist das Hydrogenase-System soweit aktiviert, daß eine schwache Nitritreduktion beobachtet werden kann. Mit längerer Adaptationszeit wird die Nitritreduktion immer stärker, wie aus den Abb. 1 und 2 ersichtlich ist.

${ }^{6}$ K. Damaschke, Z. Naturforschg. 12 b, 155 [1957].

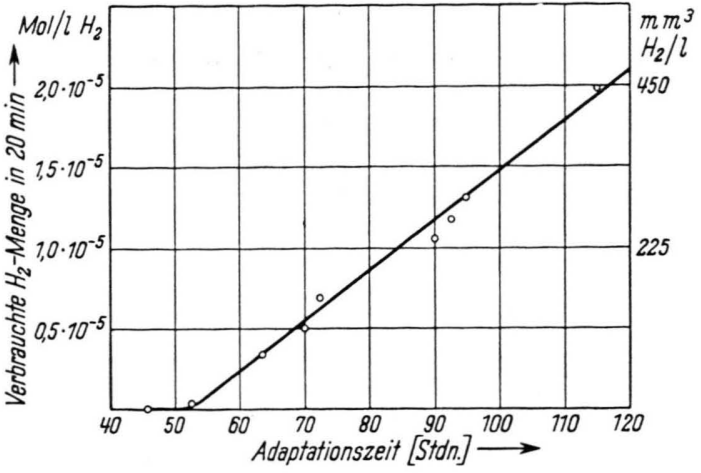

Abb. 2. Abhängigkeit der Nitritreduktion von der Adaptationszeit. Nitritmenge jeweils $2 \cdot 10^{-4} \mathrm{Mol} / l \mathrm{KNO}_{2}$.

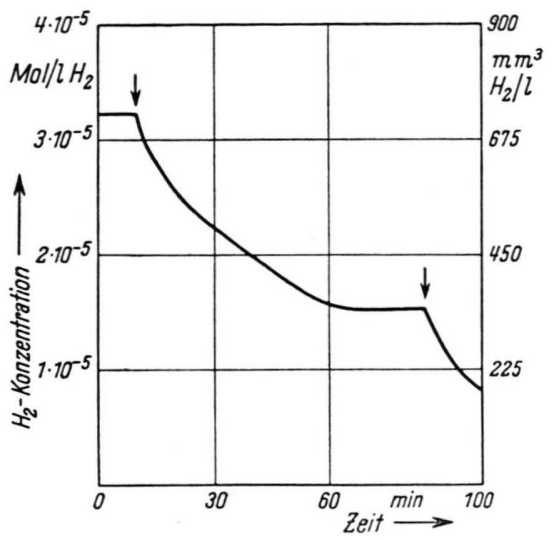

Abb. 3. Verlauf der $\mathrm{H}_{2}$-Abnahme bei Nitritzusatz. Beim Pfeil jeweils Zugabe von $1 \cdot 10^{-4} \mathrm{Mol} / l \mathrm{KNO}_{2}$. Die Adaptationszeit betrug 73 Stunden.

Abb. 3 zeigt, daß der $\mathrm{H}_{2}$-Abfall nach der Nitritzugabe zuerst schnell verläuft und dann immer langsamer wird, bis er schließlich ganz aufhört. Bei erneuter Nitritzugabe setzt wieder ein kräftiger $\mathrm{H}_{2}$-Verbrauch ein.

Diese Ergebnisse sowie uns re früheren Beobachtungen der $\mathrm{H}_{2}$-Gärung und der $\mathrm{CO}_{2}$-Reduktion mit molekularem $\mathrm{H}_{2}$ deuten darauf kin, daß auch Chlorella pyrenoidosa ein Hydrogenase-System besitzt, zu dessen Aktivierung es jedoch einer Adaptationszeit von über 2 Tagen bedarf. 